\title{
Exploring Multiwavelength AGN Variability with Swift Archival Data
}

\author{
Jonathan Gelbord ${ }^{* a}$ Caryl Gronwall, ${ }^{b}$ Dirk Grupe ${ }^{c}$ Dan Vanden Berk ${ }^{d}$ and Jian Wu ${ }^{e}$ \\ ${ }^{a}$ Spectral Sciences Inc., 4 Fourth Ave., Burlington MA 01803, USA \\ ${ }^{b}$ Department of Astronomy and Astrophysics, Pennsylvania State University, 525 Davey Lab, \\ University Park PA 16802, USA \\ ${ }^{c}$ Department of Earth and Space Sciences, Morehead State University, 235 Martindale Drive, \\ Morehead KY 40351, USA \\ ${ }^{d}$ Department of Physics, St. Vincent College, 300 Fraser Purchase Rd, Latrobe PA 15650, USA \\ ${ }^{e}$ College of Information Sciences and Technology, Pennsylvania State University, 322 IST, \\ University Park PA 16802, USA \\ E-mail: jgelbordespectral.com, jxw394@ist.psu.edu
}

\begin{abstract}
We are conducting an archival Swift program to measure multiwavelength variability in active galactic nuclei (AGN). This variability information will provide constraints on the geometry, physical conditions and processes of the structures around the central black holes that emit and reprocess the observed flux. Among our goals are: (1) to produce a catalog of type 1 AGN with time-resolved multi-wavelength data; (2) to characterize variability in the optical, UV and $\mathrm{X}$-ay bands as well as changes in spectral slope; (3) to quantify the impact of variability on multiwavelength properties; and (4) to measure correlated variability between bands. Our initial efforts have revealed a UVOT calibration issue that can cause a few percent of measured UV fluxes to be anomalously low, by up to $30 \%$.
\end{abstract}

Swift: 10 Years of Discovery,

2-5 December 2014

La Sapienza University, Rome, Italy

\footnotetext{
${ }^{*}$ Speaker.
} 


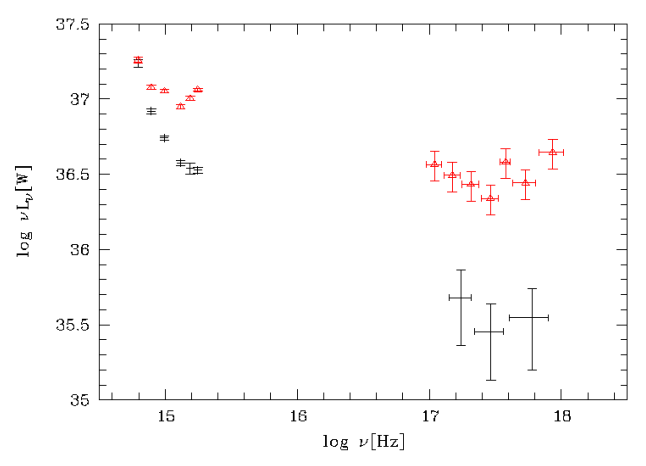

Figure 1: Optical through X-ray SEDs for 1RXS J2349-31 as measured by Swift in September 2006 (black) and May 2007 (red). The X-ray flux increased tenfold whereas the optical-UV fluxes rose by no more than a factor of three, causing $\alpha_{\mathrm{ox}}$ to change from -1.50 to -1.25 in eight months [5].

\section{Introduction}

\subsection{Multiwavelength variability in AGN}

Active Galactic Nuclei (AGN) are intrinsically broad-band beasts, with emission spanning the electromagnetic spectrum [e.g., 4]. Emission in different wavebands is in general dominated by different structures. For instance, the optical-to-ultraviolet (UV) emission is dominated by the big blue bump (BBB), which is the integrated black body emission from regions of the accretion disk with a distribution of temperatures, whilst X-rays are thought to be due to inverse Compton (IC) scattering (likely the upscattering of BBB photons by a coronal region around the accretion disk), and the infrared (IR) band exhibits thermal emission from both AGN-heated dust and the host galaxy stellar population. Consequently, a complete picture of AGN requires multiwavelength data. When parts of the spectral energy distribution (SED) are not observed, a generic spectral shape is assumed to estimate the unobserved flux.

AGN emission is also variable, with intrinsic time scales that may differ for each emitting region. The most compact regions, generally the energetic regions closest to the central black hole, can vary the most rapidly. Consequently, the temporal behavior can differ across the SED. If the timescale or amplitude of variations differ between wavebands, this will unavoidably influence multiwavelength measurements such as the optical to X-ray spectral index, $\alpha_{\mathrm{ox}}$ (defined as $f_{v} \propto v^{+\alpha}$; effectively the relative power of the accretion disk and the IC emission). As a result, a single measurement may not provide a typical value for a given AGN (e.g., Figure 1, in which the $\alpha_{\mathrm{ox}}$ of 1RXS J2349-31 is shown to change by +0.25 within a span of eight months). Variability therefore adds noise to measurements of multiwavelength parameters, which will weaken or obscure correlations that could provide insight into the properties and processes of AGN.

Historically, the availability of simultaneous multiwavelength data has been limited due to the difficulty of coordinating observations. As a result, non-simultaneous data have often been combined to establish multiwavelength parameters. The danger of this is demonstrated by 1RXS J2349-31 (Figure 1): if the optical measurements of one epoch are combined with the X-ray measurements of the other, the $\alpha_{\mathrm{ox}}$ values obtained would be either -1.75 or -1.00 , whereas measurements from simultaneous data only show a change from -1.50 to -1.25 . Instances such as 


\begin{tabular}{ccc} 
Min. num. of days with data & Swift fields & $X M M$ fields \\
\hline 2 & 5000 & 736 \\
20 & 350 & 6 \\
50 & 150 & 0 \\
100 & 44 & 0
\end{tabular}

Table 1: Tallies of fields that were re-observed on multiple days by Swift and XMM. For both observatories, the maximum area per field with simultaneous X-ray and UV or optical data is $17^{\prime} \times 17^{\prime}$. Swift data are as of 13 May 2012 and XMM data are based on the 2XMM-DR3 catalog [11].

this amplify the impact of variability upon multiwavelength parameter measurements when nonsimultaneous data are combined. For example, an anti-correlation has been established between UV luminosity ( $\mathrm{L}_{\mathrm{UV}}$ ) and $\alpha_{\mathrm{ox}}$ [e.g., 6]. We have shown that the scatter in this correlation is reduced by at least $20 \%$ when only simultaneous UV and X-ray data are used [12].

\subsection{Why Swift?}

The data archive of the Swift satellite is a unique resource for studies of multiwavelength variability. By default, Swift collects data simultaneously with the X-ray Telescope (XRT) and the Ultraviolet/Optical Telescope (UVOT). The availability of simultaneous data in these bands is critical, as these are the bands in which radio-quiet AGN vary most strongly.

The XRT and UVOT have wide, co-aligned fields of view, providing nearly 300 sq. arcmin of overlapping area with each pointing. This large area provides opportunities to measure many serendipitous sources as well as the intended targets of each observation. As of 13 May 2012, the Swift archive included 667 sq. degrees of sky covered by both instruments with exposure times of at least $1 \mathrm{ks}$ and $222 \mathrm{sq}$. degrees with at least $10 \mathrm{ks}$ of exposure time. In addition, there are many fields with repeated observations, making it possible to measure variability on time scales ranging from hours to years. This includes 400 sq. degrees with data taken at least one day apart.

$X M M$-Newton also offers simultaneous X-ray, UV and optical data. In contrast to Swift, which is in a 1.6 hour orbit and cannot observe any point in the sky for more than $\sim 40$ min per orbit, $X M M$ is capable of observing a single field continuously for more than a day, so it is better suited for measuring variability behavior on intraday timescales. However, for timescales of days to years, Swift provides unparalleled temporal coverage, with vastly more repeated fields and higher cadence monitoring (Table 1).

\section{The catalog}

We are currently building a catalog of AGN with multiple multiwavelength observations in the Swift archive. The sample will be limited to spectroscopically-confirmed AGN with secure classifications, redshifts and means of estimating parameters such as their black hole masses $\left(\mathrm{M}_{\mathrm{BH}}\right)$ and Eddington fractions $\left(\mathrm{L} / \mathrm{L}_{\mathrm{Edd}}\right)$. We will compile X-ray data and photometry from six UVOT filters. The catalog will include time-resolved flux values, upper limits for epochs with non-detections, spectral indices and hardness ratios, and estimates of the fluxes in fixed bands in the emitted frame.

We anticipate having 2000-4000 AGN in the final sample. This will include about 2000 SDSS QSOs from the SDSS-DR7, -DR9 and -DR10 quasar catalogs [10, 8, 9] and up to 2000 additional 
AGN appearing in the literature. Of order 1000 of the sample members will be purely-random, serendipitously-observed sources. The sample will represent a broad range of intrinsic properties (such as luminosities, black hole masses and accretion rates) and will be large enough to subdivide by these parameters in order to assess how they affect the variability behavior.

\section{Objectives}

The primary goals of this program may be summarized as follows:

- To build the largest catalog of AGN with simultaneous X-ray, optical and UV data.

- To use these data to obtain cleaner measurements of multiwavelength parameters, with which we will refine established correlations and test for new ones.

- To quantify the flux and spectral variability on timescales from hours to years, characterizing both individual objects and average behavior amongst subsamples defined by various intrinsic properties.

- For a limited number of AGN with both intensive monitoring and high signal-to-noise ratio data, we will put constraints on any correlated variability between the bands.

Taken together, these efforts will quantify the strength and rate of incidence of spectral variability, produce improved estimates of AGN bolometric luminosities, and provide constraints on the geometry and properties of the emission region structures. Any relationships found between observed variability and intrinsic parameters should provide insight into the underlying processes.

\section{Spurious UVOT light curve dips: a newly discovered calibration issue}

As a test case for our UVOT data processing scripts, we produced light curves for the AGN NGC 5548 [7, 3]. In Figure 2, we present a section of these light curves during which each observation included data in all six filters. A striking feature of these curves is a set of abrupt, short-lived flux dips. These apparent flux drops are (1) seen in simultaneous data across multiple filters; (2) strongest at short wavelengths (by up to $34 \%$ in UVW2, 22\% in UVM2 and 17\% in UVW1); (3) weak or absent in the optical bands; (4) found to exceed $10 \%$ in $4.5 \%$ of UVW2 measurements, $3.3 \%$ of UVM2, $1.0 \%$ of UVW1 and never in the optical filters; (5) sporadic, not periodic; and (6) not seen in the light curves of any field stars.

Upon first inspection, the light curve dips look real. There are no obvious defects in the data (no image artifacts, elevated backgrounds, tracking errors, known bad pixels, etc.). Moreover, no other objects in the field are affected when the AGN dips. However, the short timescales are physically implausible (extreme examples include a 33\% drop in 29 min and a $20 \%$ drop in just $19 \mathrm{~min}$; in both instances, fluxes measured hours later were consistent with the levels before the drops). Additionally, no comparable dips are found in contemporaneous UV data from HST [2].

By comparing measured fluxes to neighboring points in the light curve, we have identified 85 dips [3]. Projecting these back into raw detector coordinates, we found that almost all are tightly clustered within a few small regions on the detector (Figure 3, left panel). It appears that these 


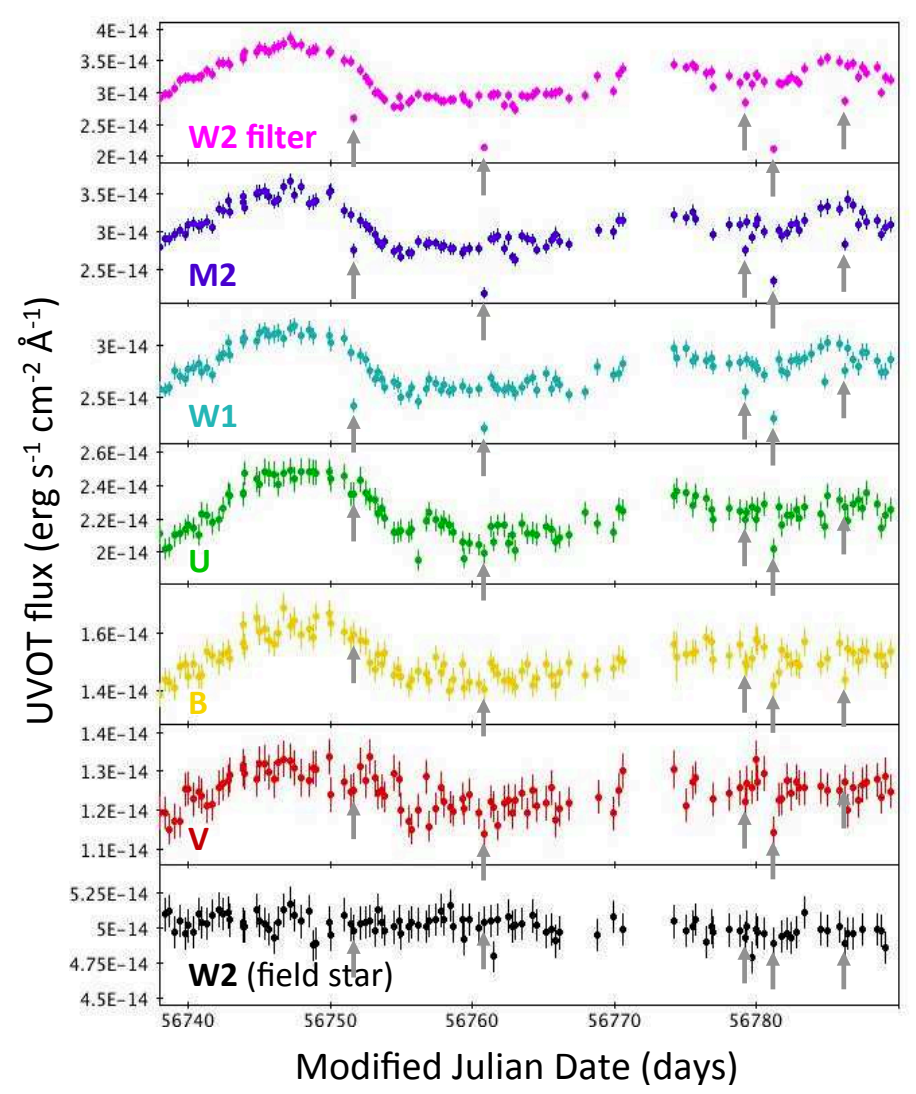

Figure 2: UVOT light curves for NGC 5548 in six filters (increasing in wavelength from the top panel down). For comparison, the bottom panel presents the W2 light curve of a field star. Grey arrows indicate measurements from five observations in which the AGN light curve exhibits clear dips in the UV filters.

are detector locations at which the sensitivity is reduced, especially at short wavelengths. While this effect has not been noted previously, these regions line up with features that can be seen in the source-subtracted background images presented by Breeveld et al. ([1]; Figure 3, right panel).

In our analysis of the dips in the NGC 5548 light curve, we define regions within which the detector sensitivity appears to be reduced (see [3] for details). These preliminary regions are adequate to clean up the NGC 5548 light curves, but the data from this AGN alone are insufficient to precisely define all areas of reduced sensitivity; portions of the defined regions may not have reduced sensitivity whilst there may be other problematic areas that were not probed by the NGC 5548 data. However, the fact that the present regions cover $4 \%$ of the central $5^{\prime} \times 5^{\prime}$ of the UVOT field of view suggests that a few percent of all UVOT measurements may be affected.

\section{Acknowledgements}

We gratefully acknowledge the support from NASA under award NNH13CH61C.

\section{References}

[1] Breeveld, A. A., et al., 2010 Further Calibration of the Swift Ultraviolet/Optical Telescope, MNRAS 406, 1687. 


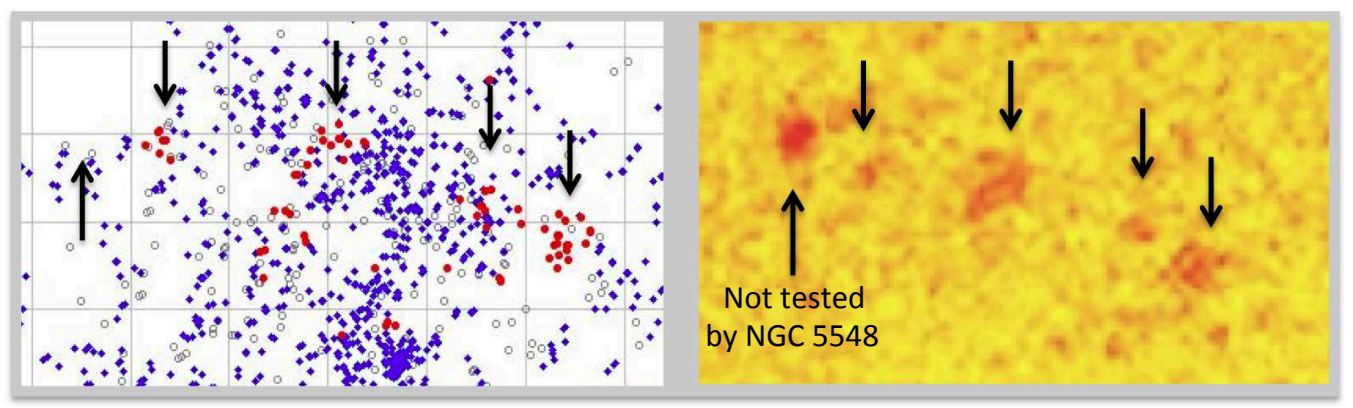

Figure 3: A comparison of the spatial distribution of NGC 5548 flux outliers to blemishes present in a source-subtracted background image in the UVOT UVM2 filter. At left, we display a map of NGC 5548 measurements in raw UVOT detector coordinates (spanning roughly from X,Y $=295,505$ to 620,710 in a frame with $1024 \times 1024$ pixels [i.e., the default $2 \times$ binning]). Red points are flux dips, blue points are nondipping measurements and open circles are measurements for which the dipping test was not applied because of insufficient data within \pm 2 days. At right is a source-subtracted background image in the UVOT M2 filter covering the same detector region [1]. This image exhibits dark patches where the measured background flux is lower. The locations of these blemishes are indicated by arrows in both panels.

[2] De Rosa, G., et al., 2015 Space Telescope and Optical Reverberation Mapping Project. I. Ultraviolet Observations of the Seyfert 1 Galaxy NGC 5548 with the Cosmic Origins Spectrograph on Hubble Space Telescope, Submitted to ApJ (arXiv:1501.05954) .

[3] Edelson, R., et al., 2015 Space Telescope and Optical Reverberation Mapping Project. II. Swift and HST Reverberation Mapping of the Accretion Disk of NGC 5548, Submitted to ApJ (arXiv:1501.05951).

[4] Elvis, M., et al., 1994 Atlas of Quasar Energy Distributions, ApJS 95, 1.

[5] Grupe, D., et al., 2010 The Simultaneous Optical-to-X-ray Spectral Energy Distribution of Soft X-ray Selected Active Galactic Nuclei Observed by Swift, ApJS 187, 64.

[6] Just, D.W., et al., 2007 The X-Ray Properties of the Most Luminous Quasars from the Sloan Digital Sky Survey, ApJ 665, 1004.

[7] McHardy, I. M., et al., 2014 Swift Monitoring of NGC 5548: X-ray Reprocessing and Shortterm UV/Optical Variability, MNRAS 444, 1469.

[8] Pâris, I., et al., 2012 The SDSS Quasar Catalog: Ninth Data Release, A\&A 548, 66.

[9] Pâris, I., et al., 2014 The SDSS Quasar Catalog: Tenth Data Release, A\&A 563, 54.

[10] Schneider, D.P., et al., 2010 The SDSS Quasar Catalog. V. Seventh Data Release, AJ 139, 2360.

[11] Watson, M., et al., 2009 The XMM-Newton serendipitous survey. V. The Second XMM-Newton serendipitous source catalogue, A\&A 493, 339.

[12] Wu, J., et al., 2012 A Quasar Catalog with Simultaneous UV, Optical, and X-Ray Observations by Swift, ApJS 201, 10. 